\title{
LES QUESTIONS BARBARES DE PLUTARQUE: UN ESSAI DE RECONSTITUTION
}

La recherche des causes, ou étiologie, occupe une place prépondérante dans l'œuvre et dans la pensée de Plutarque. Sans doute assez normale chez un philosophe rompu au genre des problemata, elle témoigne surtout d'une curiosité de tous les instants pour le monde qui l'entoure. Sans même parler des traités dans lesquels il s'interroge, par exemple, sur les causes des délais de la justice divine (De sera numinis vindicta), sur les raisons du déclin des oracles à son époque (De defectu oraculorum), sur la signification de l'Epsilon de Delphes (De E apud Delphos) ou sur la présence du visage qu'on voit dans la lune (De facie quae in orbe lunae apparet), nous avons conservé de Plutarque plusieurs œuvres sous la forme de questions-réponses dans lesquelles le Chéronéen laisse libre cours à ses interrogations sur les origines de toutes sortes de phénomènes, de croyances, de pratiques, d'institutions, d'expressions et d'autres sujets : ce sont, en particulier, les Questions

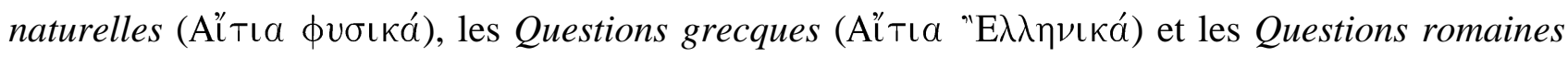
(Aïtıa "P

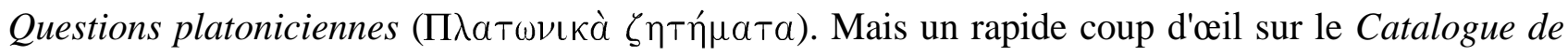
Lamprias (une liste antique des ouvrages de Plutarque datant vraisemblablement du III ${ }^{\mathrm{e}}-\mathrm{IV}^{\mathrm{e}}$ s.) ${ }^{1}$ révèle que Plutarque avait rédigé bien d'autres œuvres à caractère étiologique, aujourd'hui perdues ${ }^{2}$ : à côté de traités sur les comètes (no. 99), sur les tremblements de terre (no. 212), sur les fondations de villes (no. 195) ou sur les théories scientifiques (no. 196), on en compte plusieurs qui portent explicitement le titre de Aitíaı, notamment les Questions sur les «Signes célestes » d'Aratos

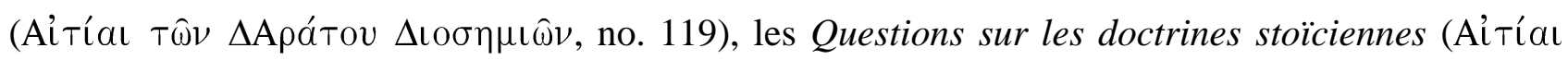

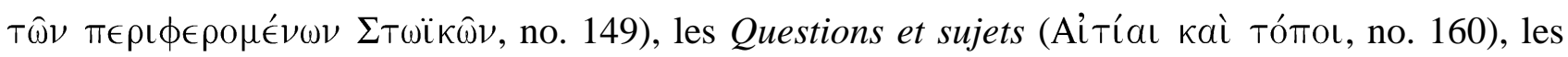

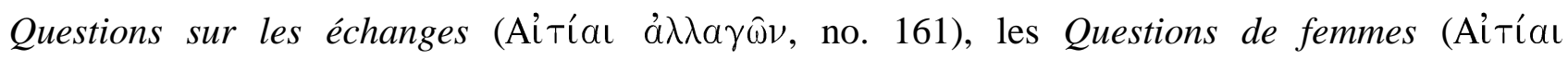

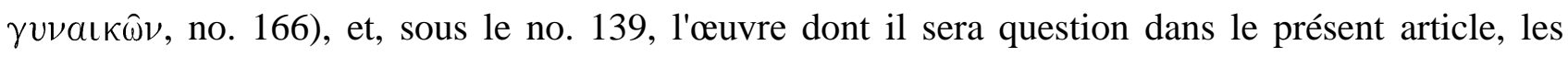

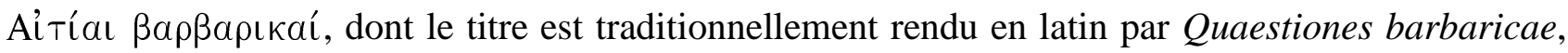

\footnotetext{
${ }^{1}$ Pour une présentation du Catalogue de Lamprias, voir K. Ziegler, Plutarchos von Chaironeia, Stuttgart, 1949 (= Realencyclopädie, XXI, 1951), col. 696-702 et surtout J. Irigoin, « Le Catalogue de Lamprias, tradition manuscrite et éditions commentées », Revue des études grecques, 99, 1986, p. 318-331. L'édition la plus récente est celle de J. Irigoin, Plutarque, Oeuvres morales, tome I, $1^{\text {ère }}$ partie, Paris, CUF, p. CCCXI-CCCXVIII, qui complète celle de F.H. Sandbach, Plutarchi Moralia, vol. VII, Leipzig, Teubner, 1967, p. 1-10, reprise par le même auteur dans Plutarch's Moralia, vol. XV : Fragments, London-Cambridge, MA (Loeb), 1969, p. 8-29.

${ }^{2}$ Une liste commode des œuvres étiologiques de Plutarque est fournie par G.W.M. Harrison, « Problems with the Genre of Problems: Plutarch's Literary Innovations », Classical Philology, 95.2, 2000, p. 193-199; voir aussi, dans le présent volume, la contribution de Thierry Grandjean, «Le recours à l'étiologie chez Dion de Pruse et chez Plutarque de Chéronée », p. 147.
} 
c'est-à-dire les Questions barbares, et qui devait manifestement constituer le pendant des Questions grecques (no. 166) et des Questions romaines (no. 138), toutes deux conservées ${ }^{3}$. Quiconque s'intéresse aux barbares chez Plutarque ne peut que déplorer la perte de cet ouvrage et se poser la question suivante : peut-on espérer en reconstituer le contenu? Tel est le sujet des réflexions qui vont suivre ${ }^{4}$.

Avant d'y répondre, il convient toutefois de poser deux autres questions fondamentales : cette œuvre a-t-elle jamais été écrite et, si oui, est-elle de Plutarque? La première interrogation est justifiée dans la mesure où le Catalogue de Lamprias n'est pas la plus fiable des sources, puisqu'il omet de mentionner de nombreuses œuvres de Plutarque qui sont pourtant conservées et qu'à l'inverse il en recense un grand nombre dont on n'a aucune autre trace par ailleurs ${ }^{5}$. C'est le cas des Questions barbares, qui ne sont mentionnées nulle part, ni chez Plutarque, ni chez aucun autre auteur. Malgré ces réserves, nous n'avons pas plus de raisons de douter de l'existence de cette œuvre que pour n'importe quel titre figurant dans le Catalogue de Lamprias. En effet, si ce dernier, dont on pense qu'il a pu être le catalogue d'une bibliothèque, mentionne des Questions barbares, c'est que cet écrit a manifestement existé à une certaine époque. Mais est-il de Plutarque? Là encore, la question est justifiée, puisque même la paternité des Questions grecques, qui sont pourtant conservées, a été mise en doute, sous prétexte qu'une telle œuvre serait indigne de Plutarque ${ }^{6}$.

\footnotetext{
${ }^{3}$ Les Questions grecques et les Questions romaines ont récemment connu un regain d'intérêt, surtout de la part de chercheurs français. Il convient en particulier de signaler la nouvelle édition de J. Boulogne, Plutarque, Oeuvres morales, tome IV, Paris, CUF, 2002, son important article «Les"Questions romaines" de Plutarque» dans Aufstieg und Niedergang der römischen Welt, II, 33.6, 1992, p. 4682-4708, et son analyse détaillée dans Plutarque, un aristocrate grec sous l'occupation romaine, Lille, 1994, p. 75-146, ainsi que ses deux articles « Le sens des Questions romaines de Plutarque », Revue des études grecques, 100, 1987, p. 471-476, et «La structuration du temps dans les Étiologies romaines de Plutarque », Euphrosyne n.s. 28, 2000, p. 231-236; par ailleurs, deux remarquables volumes collectifs : P. Payen (éd.), Plutarque: Grecs et Romains en Questions, Entretiens d'archéologie et d'histoire, Saint-Bertrand-deComminges, 1998 ; M. Nouilha, J.-M. Pailler, P. Payen (edd.), Plutarque. Grecs et Romains en parallèle, Paris, 1999. Non moins précieux sont les commentaires détaillés de H.J. Rose, The Roman Questions of Plutarch, Oxford, 1924, et de W.R. Halliday, The Greek Questions of Plutarch, Oxford, 1928, et l'article de R. Preston, « Roman questions, Greek answers : Plutarch and the construction of identity », dans S. Goldhill (ed.), Being Greek under Rome. Cultural Identity, the Second Sophistic and the Development of Empire, Cambridge, 2001, p. 86-119. Enfin, signalons également l'édition espagnole de M. Lopez Salva, Plutarco. Obres Morales y de Costumbres, V, Madrid, 1989.

4 À notre connaissance, aucune reconstitution de ce genre n'a été tentée, mis à part quelques remarques très générales de Rose (The Roman Questions..., p. 49), reprises par Ziegler (Plutarchos..., col. 858, n. 1) : Rose estime que les Questions barbares étaient une sélection de notes de lecture faites à l'occasion de la rédaction de la Vie d'Artaxerxès, du traité Sur Isis et Osiris et d'autres écrits du même genre, et que les fr. 133 et 135 rassemblés par G.N. Bernardakis (Plutarchi Chaeronensis Moralia, vol. VII, Leipzig (Teubner), 1896, p. 166) [= fr. 212 et 213 dans Sandbach, Plutarch's Moralia, XV, p. 386-387] pourraient en avoir fait partie, alors que Quaest. conv. IV, 5 (= Mor. 669E-671C) et Conj. praec. 35 (= Mor. 143A-B) semblent reprendre du matériel issu de cette œuvre. Voir notre discussion ci-après.

${ }^{5}$ Selon le décompte de Ziegler (Plutarchos..., col. 701-702), sur les 227 titres du Catalogue de Lamprias, 129 ne sont pas conservés ou attestés ailleurs ; en revanche, 18 oeuvres conservées et 15 autres connues par la tradition indirecte n'y figurent pas; voir aussi Irigoin, « Le Catalogue... », p. 319.

${ }^{6}$ Cet avis a notamment été exprimé avec virulence par J.J. Hartman, De Plutarcho scriptore et philosopho, Leyde, 1916, p. 139-140 (= «Ad Plutarchi Moralia annotationes criticae», Mnemosyne n.s. 41, 1913, p. 216-217), mais rejeté par Halliday, The Greek Questions..., p. 13, par Ziegler, Plutarchos..., col. 862-863, et par tous les éditeurs modernes.
} 
Cependant, la communis opinio veut aujourd'hui, à juste titre sans doute, que les Questions grecques soient bien de Plutarque et, pour ce qui est des Questions barbares, plusieurs raisons font pencher la balance dans le même sens. En effet, il convient de rappeler que Plutarque s'est intéressé de près aux barbares, comme en témoignent sa Vie d'Artaxerxès, son Banquet des Sept Sages ainsi que son traité Sur Isis et Osiris, sans compter les très nombreuses mentions de barbares ailleurs dans les Vies et dans les Moralia ${ }^{7}$. Il ne faut pas oublier non plus qu'il existait, dans l'Antiquité, un grand nombre d'œuvres consacrées aux barbares, parmi lesquelles figurent en particulier les Nó $\mu \iota \mu \alpha \beta a \rho \beta a \rho є \alpha ́$ d'Aristote. Or, quand on sait que les fameuses Constitutions d'Aristote ont sans doute été la source principale de Plutarque pour les Questions grecques $^{8}$, il est parfaitement plausible de penser que la

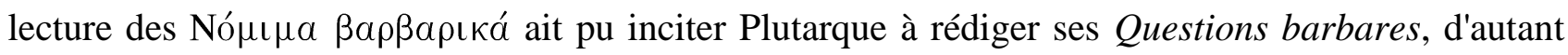
plus que ces dernières constituaient, avec les Questions grecques et les Questions romaines, «un triptyque qui renvoie au monde tel que le concevait un Grec depuis l'entrée en scène des Romains. ${ }^{9}$ Du reste, comme le note Jean Sirinelli à propos de ce type de traités, «c'était la mode des collections de citations, de pensées ou de faits marquants. (...) Ce n'était pas une tâche mineure et indigne d'un écrivain de qualité mais même, à une époque où les bibliothèques demeuraient rares, un instrument intellectuel très prisé. » ${ }^{10}$ Les rares personnes à faire mention des Questions barbares dans leurs travaux s'accordent, en tout cas, pour reconnaître en Plutarque l'auteur de cette œuvre ${ }^{11}$.

Pour en revenir à notre question initiale, il est évident que le parallèle avec les Questions grecques et les Questions romaines peut nous aider à cerner les caractéristiques principales des

\footnotetext{
${ }^{7}$ Voir nos études Plutarque et les barbares. La rhétorique d'une image, Louvain-Namur, 1999 ; «Plutarch's Timeless Barbarians and the Age of Trajan», dans P. Stadter, L. Van der Stockt (edd.), Sage and Emperor: Plutarch, Greek Intellectuals, and Imperial Power in the Age of Trajan, Louvain, 2002, p. 57-71; «Barbarians in Plutarch's Political Thought », dans: L. de Blois et al. (edd.), The Statesman in Plutarch's Works, Volume I : Plutarch's Statesman and His Aftermath : Political, Philosophical, and Literary Aspects, Leiden-Boston, 2004, p. 227-235.

${ }^{8}$ Voir notamment K. Giessen, «Plutarch's Quaestiones Graecae und Aristoteles Politien », Philologus, 60, 1901, p. 446-471 ; Halliday, The Greek Questions...,, p. 14-15; Ziegler, Plutarchos..., col. 862; Boulogne, Plutarque... (CUF), p. 181; Nouilhan-Pailler-Payen, Plutarque..., p. 29-30. Comme il ressort clairement de ces études, il est évident que les sources de Plutarque ne se limitent pas aux Constitutions d'Aristote, mais sont au contraire très variées, comme c'est le cas également des Questions romaines (à ce sujet, voir en particulier L. Van der Stockt, «Plutarch's Use of Literature. Sources and Citations in the Quaestiones Romanae », Ancient Society, 18, 1987, p. 281-292).

${ }^{9}$ C. Darbo-Peschanski, «Pourquoi chercher des causes aux coutumes? (Les Questions romaines et les Questions grecques de Plutarque)», dans P. Payen (éd.), Plutarque: Grecs et Romains en Questions, Entretiens d'archéologie et d'histoire, Saint-Bertrand-de-Comminges, 1998, p. 23. L'idée d'un " triptyque ", c'est-à-dire d'une œuvre à trois volets, est la plus communément admise. Toutefois, P. Payen ("Rhétorique et géographie dans les Questions romaines et Questions grecques de Plutarque » dans P. Payen (éd.), Plutarque: Grecs et Romains en Questions... p. 39 et 49) propose de considérer les Questions grecques et les Questions romaines comme un recueil unique, ce qui n'est pas, selon lui, en contradiction avec l'existence d'un recueil de Questions barbares.

${ }^{10}$ J. Sirinelli, Plutarque, un philosophe dans le siècle, Paris, 2000, p. 365.

${ }^{11}$ Voir Rose, The Roman Questions..., p. 48-49 ; Halliday, The Greek Questions..., p. 13 ; Ziegler, Plutarchos..., col. 858 ; Darbo-Peschanski, «Pourquoi chercher... », p. 23 ; Payen, «Rhétorique et géographie... », p. 39 n. 26 et p. 49 ; Nouilhan-Pailler-Payen, Plutarque..., p. 9; J. Boulogne, Plutarque..., CUF, p. 183; idem, «Les "Questions romaines"...», p. 4684 n. 14 ; Harrison, « Problems... », p. 194; Preston, «Roman questions... », p. 93.
} 
Questions barbares, à commencer par le titre. Le Catalogue de Lamprias le donne sous la forme

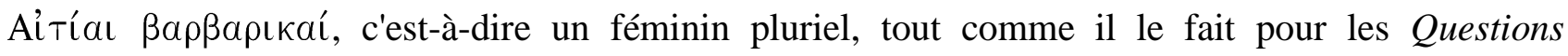

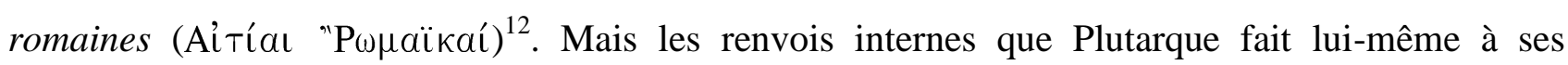
Questions romaines prouvent hors de tout doute que l'œuvre circulait sous le titre de Al̈tıa "P forme pour les Questions romaines et, par analogie, pour les Questions grecques ${ }^{13}$. Il semble dès lors préférable de retenir le neutre également pour le titre des Questions barbares, à savoir Al̈tıa

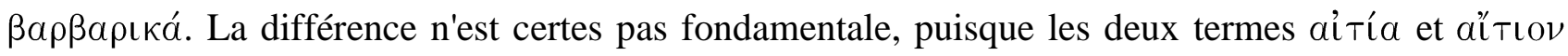
signifient à l'origine «la cause », mais le neutre a pris un sens technique d'«explication » plus conforme à la nature des traités de Plutarque. Jacques Boulogne a d'ailleurs proposé de traduire ces titres par «Étiologies » plutôt que par «Questions » comme on le fait habituellement par décalque du latin; la suggestion est excellente et mériterait d'être retenue, si elle n'avait pas contre elle le poids de la tradition séculaire qui connaît ces œuvres sous le titre de «Questions » ${ }^{14}$.

Le parallèle avec les Questions grecques et les Questions romaines nous permet également de déduire la forme sous laquelle se présentaient, selon toute vraisemblance, les Questions barbares. La typologie des Questions grecques et des Questions romaines a été bien mise en lumière dans les études détaillées mentionnées précédemment ${ }^{15}$. Les Questions romaines sont constituées de 113 questions, invariablement introduites (à deux exceptions près) par la formule Sı ̀̀ Tí («pourquoi »), alors que du côté des Questions grecques, ce sont 59 questions seulement, mais recourant à des formulations beaucoup plus variées. Quant aux réponses, dans le cas des Questions romaines, elles prennent majoritairement la forme d'hypothèses dont le nombre peut varier de une à six et qui sont formulées sur un mode interrogatif: «est-ce parce que? ou parce que? ou bien plutôt parce que? »

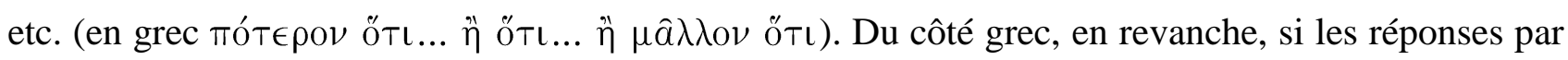
hypothèses sont certes toujours présentes, Plutarque leur préfère nettement des explications sous forme de narrations. On a cherché à expliquer ces différences par le fait que Plutarque, plus au fait

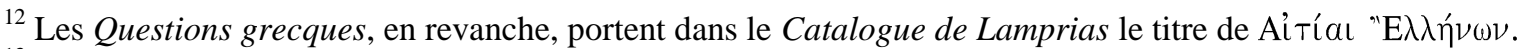

13 Voir à ce sujet les discussions de Hartman, De Plutarcho..., p. 132-136 (= Mnemosyne n.s. 41, 1913, p. 209-213); Boulogne, Plutarque..., CUF, p. 91-92 et p. 179.

${ }^{14}$ Boulogne, «Les "Questions romaines"... », p. 4683 n. 9 ; idem, Plutarque, un aristocrate..., p. 76 ; idem, Plutarque..., CUF, p. 92; idem, «Les étiologies romaines : une herméneutique des moeurs à Rome », dans P. Payen (éd.), Plutarque: Grecs et Romains en Questions, Entretiens d'archéologie et d'histoire, Saint-Bertrand-de-Comminges, 1998, p. 31. Dans le même sens, Harrison («Problems... », p. 195) propose " explanations " comme traduction la plus appropriée. Payen, («Rhétorique et géographie...», p. 65 n. 3) préfère comme nous l'appellation traditionnelle de Questions. Sur la

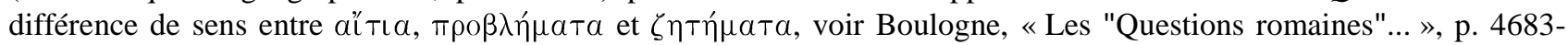
468 ; Harrison, « Problems.. .», p. 194-197 ; Darbo-Peschanski, « Pourquoi chercher... », p. 21-23.

${ }^{15}$ Outre les analyses de Boulogne, voir en particulier Nouilhan, Pailler, Payen, Plutarque..., p. 30-39 (avec de très utiles tableaux de synthèse) et Payen, « Rhétorique et géographie... », p. 39-49.
} 
des réalités grecques, pouvait en donner des explications uniques et définitives, alors que pour le monde romain, il a préféré présenter toutes les hypothèses qu'il avait trouvées dans ses sources, laissant à ses lecteurs le soin de décider laquelle était la plus plausible ${ }^{16}$. Il est dès lors difficile de savoir quel modèle suivaient les Questions barbares : on serait tenté de penser que c'était le modèle « romain » à plusieurs hypothèses ${ }^{17}$, pour autant que les sources dont disposait Plutarque le lui permettaient.

Quant aux sujets abordés dans les Questions barbares, on ne peut là encore que se baser sur une comparaison avec les Questions grecques et les Questions romaines. Devant la diversité des thèmes traités, plusieurs classifications sont possibles ; par commodité, on retiendra ici celle de Michèle Nouilhan, Jean-Marie Pailler et Pascal Payen. Ainsi, pour les Questions romaines, les trois auteurs ont identifié quatre domaines principaux auxquels peuvent être rattachées les diverses questions : le rituel (66 questions), la parenté (26), les institutions (18) et le calendrier $(4)^{18}$. Ce classement est tout à fait pertinent, mais il faut savoir qu'à l'intérieur de chacune des catégories se rencontre une grande variété de questions. D'une façon générale, dans les Questions romaines, on constate une très forte présence de la sphère du religieux, qu'on ne retrouve pas de façon aussi marquée dans les Questions grecques. Celles-ci sont caractérisées par une plus grande diversité de sujets, même si l'on parvient là aussi à les classer selon trois thèmes principaux : les fondations de villes et la colonisation, les institutions et, enfin, la religion et les sanctuaires - les éléments unificateurs étant tantôt la langue, tantôt la polis ${ }^{19}$. Quant aux réponses, on peut, avec Jacques Boulogne, les classer selon six types de causalité : la caractériologie, l'éthique, l'étymologie, l'histoire, la mythologie et la physique ${ }^{20}$.

Malgré les différences qui existent entre les Questions grecques et les Questions romaines et en dépit de la grande diversité des questions et des réponses, on peut néanmoins raisonnablement postuler que les Questions barbares devaient constituer une série de questions indépendantes, sur des sujets divers touchant de façon large les us et coutumes des barbares, en particulier leurs pratiques religieuses, leurs institutions et leur manière de vivre, et qu'à ces questions étaient

\footnotetext{
${ }^{16}$ Voir Nouilhan, Pailler, Payen, Plutarque..., p. 39 ; Boulogne, « Les "Questions romaines"... », p. 4688-4690 ; DarboPeschanski, «Pourquoi chercher... », p. 23-25; Payen, «Rhétorique et géographie... », p. 45; Preston, «Roman questions... », p. 95-97.

${ }^{17}$ C'est aussi l'avis exprimé par G.W.M. Harrison, «Tipping His Hand: Plutarch's Preferences in the Quaestiones naturales », dans L. Van der Stockt (éd.), Rhetorical Theory and Praxis in Plutarch, Louvain-Namur, 1999, p. 238 n. 4.

${ }^{18}$ Nouilhan-Pailler-Payen, Plutarque..., p. 30-34. Pour d'autres classifications, voir Boulogne, Plutarque..., CUF, p. 9293 ; idem, Plutarque, un aristocrate..., p. 77-114; Darbo-Peschanski, « Pourquoi chercher... », p. 25-28.

${ }^{19}$ Nouilhan, Pailler, Payen, Plutarque..., p. 34-35.

${ }^{20}$ Boulogne, Plutarque...,CUF, p. 93 ; idem, « Les "Questions romaines"... », p. 4696-4698.
} 
apportées une ou plusieurs réponses plus ou moins détaillées, présentées sous forme d'hypothèses successives formulées sur un mode interrogatif ou narratif ${ }^{21}$.

Si le cadre général est ainsi posé, la comparaison avec les Questions grecques et les Questions romaines n'est cependant d'aucun secours pour déterminer de façon plus concrète le contenu des Questions barbares. Et, à vrai dire, cette mission semble a priori impossible : comment, en effet, reconstituer le contenu d'une œuvre perdue? Fort heureusement, une piste de recherche nous est offerte par Plutarque lui-même : c'est qu'il avait la fâcheuse habitude de se répéter. En vérité, ce n'est pas tant qu'il se répétait, mais des recherches récentes ont révélé qu'il travaillait par fiches, qui devaient être classées par mots-clés ou par sujets et sur lesquelles se trouvaient rassemblés ce qu'on a appelé des «clusters », c'est-à-dire des regroupements d'idées ou de thèmes présentés sous une forme semi-rédigée que Plutarque pouvait ensuite utiliser à sa guise en fonction de ses besoins ${ }^{22}$. C'est ainsi que les mêmes informations se retrouvent souvent, sous une formulation très semblable, à différents endroits de son vaste corpus. C'est le cas, notamment, d'un assez grand nombre de Questions romaines et de certaines Questions grecques, dont les données sont exploitées aussi bien dans les Vies que dans les Moralia ${ }^{23}$. On peut donc faire le pari qu'il devait en être de même pour les Questions barbares et on peut par conséquent tenter de retrouver dans le corpus plutarchéen des traces de cette œuvre perdue.

Pour définir les critères de sélection, nous nous sommes inspiré des critères établis par JeanMarie Pailler pour déterminer la présence de Questions romaines dans certaines Vies de Plutarque ${ }^{24}$. Selon lui, quatre conditions doivent être réunies : $1^{\mathrm{o}}$ le passage doit être introduit ou se terminer par une interrogation ou une formule à caractère étiologique du genre «voilà pourquoi... »; $2^{\circ}$ les explications doivent porter sur des noms d'objets, de fonctions ou de lieux, ou encore l'origine et la signification de coutumes restées vivaces à l'époque de Plutarque ; $3^{\circ}$ le passage doit présenter plusieurs hypothèses plus ou moins hiérarchisées ; $4^{\circ}$ l'étiologie doit avoir des parallèles dans une ou

\footnotetext{
${ }^{21}$ Des conclusions similaires sont exprimées par A. Strobach, Plutarch und die Sprachen, Stuttgart, 1997, p. 51-52.

${ }^{22} \mathrm{Si}$ l'utilisation d'hypomnèmata par Plutarque était connue depuis longtemps, le mérite d'en avoir révélé l'ampleur et l'exact fonctionnement revient à L. Van der Stockt, voir notamment ses articles « A Plutarchan Hypomnema on Selflove », American Journal of Philology, 120, 1999, p. 575-599; «Three Aristotles equal but one Plato. On a cluster of quotations in Plutarch », dans A. Perez Jimenez, J. Garcia Lopez, R. M. Aguilar (edd.), Plutarco, Platón y Aristóteles: Actas del V Congreso Internacional de la I.P.S. Madrid-Cuenca, 4-7 de mayo de 1999. Madrid, 1999, p. 127-140;

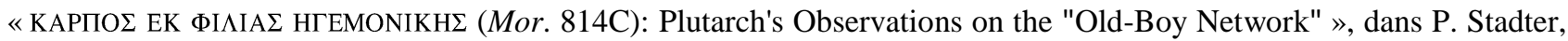
L. Van der Stockt (edd.), Sage and Emperor: Plutarch, Greek Intellectuals, and Imperial Power in the Age of Trajan, Leuven, 2002, p. 115-140.

${ }^{23}$ Voir notamment Halliday, The Greek Questions..., p. 14 ; Boulogne, Plutarque..., CUF, p. 102 n. 39 et p. $181-182$; idem, «Les "Questions romaines"...», p. 4684-468; J.-M. Pailler, «Les Questions dans les plus anciennes Vies romaines. Art du récit et rhétorique de la fondation », dans P. Payen (éd.), Plutarque: Grecs et Romains en Questions, Entretiens d'archéologie et d'histoire, Saint-Bertrand-de-Comminges, 1998, p. 77-94; Nouilhan-Pailler-Payen, Plutarque..., p. 329-333.

${ }^{24}$ J.-M. Pailler, « Les Questions... », p. 83.
} 
plusieurs des Questions romaines. Lorsque les quatre conditions sont réunies, Pailler parle d'une véritable Question; à la présence de trois conditions, d'une quasi-Question; à deux, d'ébauche de Question si le développement est court et de climat de Question si le développement est long. Si une seule condition est remplie, Pailler préfère « renoncer à toute qualification étiologique ».

Dans le cas des Questions barbares, il est évident que la quatrième condition de Pailler n'est pas applicable, puisque l'œuvre n'est pas conservée. La deuxième condition en devient d'autant plus importante ; or, comme la comparaison avec les Questions grecques et les Questions romaines l'a établi, on peut raisonnablement admettre - c'est notre postulat de base - qu'est matière à question tout sujet portant sur les pratiques religieuses, les institutions et les us et coutumes des barbares. Si ce premier critère de sélection est rempli, nous proposerons de parler $1^{\mathrm{o}}$ de Question potentielle si aucune explication n'est donnée; $2^{\circ}$ d'amorce de Question si l'explication donnée est courte ; $3^{\circ}$ d'ébauche de Question si l'explication est courte et accompagnée d'une formule introductive ou conclusive ; $4^{\circ}$ de climat de Question si l'explication est longue ; $5^{\circ}$ de quasi-Question si plusieurs explications sont fournies; $6^{\circ}$ de véritable Question si une explication longue ou plusieurs explications sont données et qu'elles s'accompagnent d'une formule introductive ou conclusive.

Les «Questions potentielles »sont évidemment les plus nombreuses - nous en avons dénombré dix-huit -, mais on ne peut pas leur accorder beaucoup d'importance dans la mesure où il s'agit simplement de sujets qui, de façon tout à fait hypothétique, auraient pu être traités dans les Questions barbares, mais pour lesquels aucune explication n'est donnée et pour lesquels nous n'avons aucun indice concret à part, précisément, le sujet ${ }^{25}$. Il s'agit simplement de pratiques curieuses, surprenantes, inhabituelles qui, à nos yeux, auraient mérité une explication, sans qu'on puisse savoir si cela a jamais été le cas. Il est donc inutile de s'y attarder et nous nous contentons d'en donner ici une liste sommaire ${ }^{26}$.

\footnotetext{
${ }^{25}$ Nous avons conscience que, de ce fait, beaucoup d'autres extraits de Plutarque pourraient sans doute être rangés parmi les «Questions potentielles ». Nous excluons toutefois les fragments 133 et 135 Bernardakis (= fr. 212-213 Sandbach), que Rose estime provenir des Questions barbares (cf. The Roman Questions..., p. 49), ainsi que le fr. 190 Sandbach; en effet, bien qu'ils traitent des origines égyptiennes de cultes et de croyances grecques, ils ne nous semblent pas correspondre à des sujets de questions. De même, alors que des pratiques barbares sont mentionnées à plusieurs endroits dans les Questions grecques et les Questions romaines (cf. Payen, « Rhétorique et géographie... », p. 44, 49, 59-60; Darbo-Peschanski, «Pourquoi chercher... », p. 23), nous n'avons retenu qu'un seul passage parmi les «Questions potentielles (voir ci-dessous).

${ }^{26}$ De superstit. 165F ( $\beta a ́ \rho \beta \alpha \rho \alpha$ какá : se vautrer dans la boue, s'enduire de fange, célébrer des sabbats, se jeter face contre terre); De superstit. 170C (les Syriens ne mangent pas de mendoles ou d'anchois) ; De superstit. 171B-D (sacrifices humains pratiqués par les Gaulois, Scythes, Carthaginois, Perses) ; Quaest. rom. 279A (les Tyriens entourent les statues de chaînes) ; De Iside 358C-D (en mémoire d'Horus, les Égyptiens jettent une corde à terre et la coupent en morceaux) ; An virt. doc. possit 440A (les Scythes crèvent les yeux des esclaves pour faire baratter le lait); An vitiositas suff. 499D (les Scythes n'ensevelissent pas leurs morts) ; De sera num. vind. 565A (les Perses font fouetter les vêtements des condamnés); Quaest. conv. 624A (Mithridate surnommé le nouveau Dionysos) ; Quaest. conv. 646E (les barbares se
} 
Dans ce que nous appelons les « amorces de Question », une explication est en revanche fournie, mais elle est toujours courte et surtout unique, si bien qu'il est impossible, là encore, de savoir si ces sujets étaient bel et bien traités dans les Questions barbares. Au moins la présence d'une explication est-elle un indice qu'une question a pu éventuellement être développée par Plutarque. Ainsi, pour ne prendre que quelques exemples parmi les douze cas que nous avons identifiés, Plutarque explique que certains barbares, en signe de deuil, descendent dans les fosses des morts et y restent plusieurs jours, refusant de voir la lumière du soleil, parce que le mort en est lui-même privé (Consol. ad Apoll. 113A-B) ; que chez les Scythes, les Hyrcaniens et les Bactriens, on laisse pourrir ou dévorer par les animaux les corps des morts, car on estime que c'est là une marque de bonheur (An vitiositas suff. 499D); que les Mèdes et les Assyriens vouent un culte au feu, car dans leur crainte superstitieuse, ils préfèrent vénérer les forces maléfiques (De facie 935B); et que les femmes égyptiennes, selon la tradition ancestrale, ne devaient pas porter de chaussures, ceci pour les forcer à rester à la maison (Conj. praec. 142C) ${ }^{27}$. Ces exemples illustrent bien pourquoi on ne peut parler que $\mathrm{d}^{\prime}$ «amorces de Question »: ils comportent certes un début d'explication, mais celle-ci reste très succincte et rien n'autorise à penser que ces questions ont vraiment été développées par Plutarque. Un indice supplémentaire serait la présence d'une formule interrogative ou conclusive, ce qui nous amènerait à parler d'une «ébauche de Question », mais nous n'avons trouvé qu'un seul passage qui réponde à ce critère : dans le traité Sur Isis et Osiris (356E), au terme de sa narration d'un épisode du mythe d'Isis, dans lequel des enfants avaient indiqué à la déesse où elle trouverait le coffre dans lequel Osiris avait été enfermé par Typhon, Plutarque conclut en disant : «C'est pour cela que les Égyptiens attribuent aux petits enfants un don de divination et tirent des présages de leur babil, surtout lorsqu'ils jouent dans des lieux consacrés en criant ce qui leur passe par la tête. ${ }^{28}$ Cette question aurait fort bien pu faire l'objet d'un développement sous forme de narration dans les

couvrent de la peau de leurs animaux au lieu de prendre leur laine) ; Quaest. conv. 649E (les riches babyloniens dorment sur des outres remplies d'eau) ; Quaest. conv. 734B (les femmes gauloises apportaient aux bains des bols de bouillie de légumes qu'elles mangeaient tout en se lavant); De soll. anim. 976B-C (les femmes égyptiennes dorment à côté de crocodiles) ; De comm. notit. 1064B (dans une peuplade éthiopienne, c'est un chien qui exerce la royauté) ; Num. 18.6 (calendrier de trois mois chez certains barbares); Artax. 3.2 (le rituel du sacre des rois perses) ; Artax. 16.3-7 (le supplice des auges) ; Artax. 26.4-5 (l'usage des rois perses selon lequel l'héritier désigné demande un présent à celui qui l'a désigné).

${ }^{27}$ Les huit autres passages sont : De adul. 50D (les flatteuses de Chypre sont appelées les « escabelles » en Syrie) ; Conj. praec. 140B (les rois perses renvoient leurs femmes au moment des beuveries) ; De Iside 355C (les Egyptiens désignent Ochos sous le nom « le Poignard»); An vitiositas suff. 499C (les veuves indiennes s'immolent par le feu avec le cadavre de leur mari) ; De sera num. vind. 557D (les Thraces marquent encore leurs femmes pour venger Orphée) ; Quaest. conv. 703E (les rois perses font servir le repas de leurs esclaves et de leurs chiens à leurs généraux et amis); De plac. phil. 911B (les Éthiopiens vieillissent rapidement et les Bretons vivent jusqu'à cent vingt ans) ; De esu carn. 996E (les Égyptiens extraient les viscères des morts et les exposent au soleil).

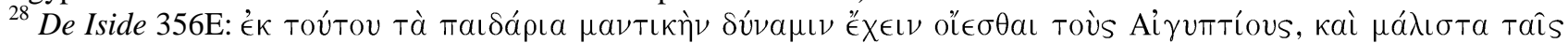

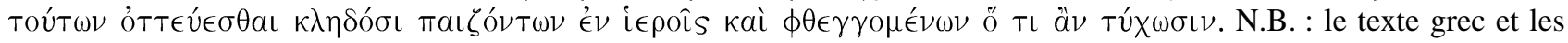
traductions de tous les extraits cités proviennent des volumes de la Collection des Universités de France. 
Questions barbares, mais les indices restent maigres et nos critères définissant les «ébauches de Question » demandent sans doute à être révisés.

Quant aux exemples qui répondent aux critères d'un «climat de Question », c'est-à-dire une matière à question développée au moyen d'une explication plus longue, nous en avons identifié trois. Dans l'un d'eux (De sera num. vind. 557D), Plutarque rapporte que les barbares de l'Éridan s'habillent de noir en signe de deuil pour Phaéthon, mais qu'à ses yeux, cette coutume est parfaitement ridicule puisque les contemporains de la mort de Phaéthon n'y avaient même pas pris garde et que ce n'est que cinq ou six générations plus tard que les gens ont commencé à changer de costume pour mener le deuil en son honneur. Cet élément de discussion critique est ce qui nous fait pencher ici en faveur d'un «climat de Question». Un autre exemple se trouve dans le traité Sur le froid primitif, dans lequel Plutarque explique pourquoi, chez les Perses, il arrivait qu'un suppliant entre dans un fleuve avec une torche allumée (De primo frig. 950F). Plus significatif, cependant, est cet extrait des Préceptes de mariage (143A-B) :

«Dans la ville de Leptis, en Libye, c'est une tradition que, le lendemain des noces, la jeune mariée envoie demander une marmite chez la mère de son mari : celle-ci ne la lui donne pas et prétend qu'elle n'en a pas, afin que, dès le début, la jeune femme apprenne à connaître le caractère de marâtre de sa belle-mère et que, si par la suite il se produit quelque heurt plus rude, elle n'en conçoive ni colère ni ressentiment. Connaissant cet état de choses, elle doit porter remède à ce qui l'occasionne : il existe une jalousie de la mère concernant l'affection de son fils pour sa bru. Le seul remède au mal, c'est qu'elle fasse naître personnellement chez son mari de l'affection pour elle sans pour autant détourner ni diminuer celle qu'il porte à sa mère. ${ }^{29}$

Les éléments qui dénotent le «climat de Question» sont les suivants : c'est une tradition, visiblement encore actuelle puisque Plutarque en parle au présent ; il s'agit d'une coutume bizarre (« demander une marmite à sa belle-mère ») ; une longue explication double est avancé : apprendre à connaître le caractère de marâtre et ne pas attiser la jalousie de la belle-mère. Il est donc très facile d'imaginer la question suivante: «Pourquoi, à Leptis, est-ce la tradition que la jeune mariée

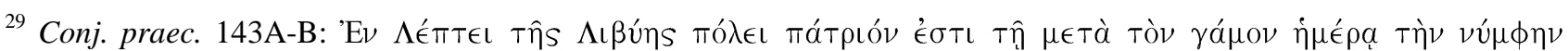

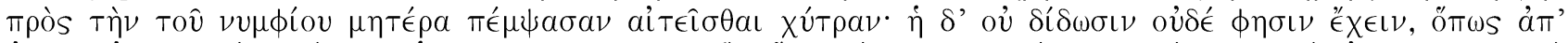

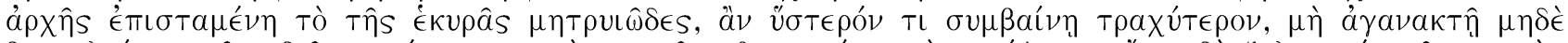

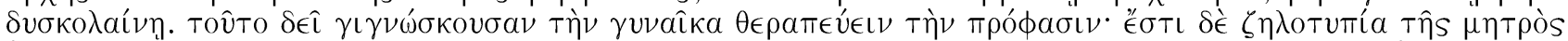

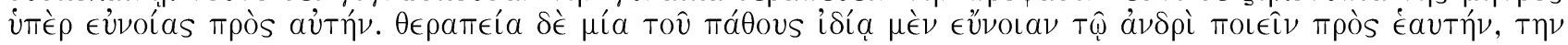

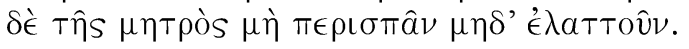


demande une marmite à sa belle-mère? » A vrai dire, il ne manque que la formulation de la question pour qu'on puisse parler d'une véritable question ${ }^{30}$.

C'est le cas également pour la catégorie des «quasi-Questions », qui présentent plusieurs explications pour un même sujet sans que la question soit formellement posée. Nous en avons identifié cinq, dont quatre proviennent du traité Sur Isis et Osiris : ainsi, Plutarque donne plusieurs raisons pour expliquer pourquoi les prêtres égyptiens s'abstiennent de poisson (353C-D), pourquoi, une fois l'an, les Égyptiens sacrifient et mangent un porc alors qu'ils s'en abstiennent habituellement (353F-354A), en quoi le culte d'Osiris peut être assimilé à celui du Soleil (372C-D) et quelle est la signification des fumigations rituelles pratiquées par les Égyptiens (383B-D). Nous ne retiendrons toutefois ici que l'exemple provenant du traité De l'envie et de la haine (537A-B), car il illustre bien en quoi consiste une quasi-question :

«En second lieu, la haine s'exerce même contre des bêtes brutes - il y a des gens qui haïssent les belettes, les cantharides, les crapauds, les serpents ; Germanicus ne supportait ni le chant ni la vue d'un coq ; les mages de Perse tuaient les rats, parce qu'eux-mêmes haïssaient cet animal, puis dans la pensée que leur dieu l'avait en aversion ; à peu près tous les Arabes et les Éthiopiens les ont en aversion. ${ }^{31}$

Nous avons ici une matière à question («les mages perses tuaient les rats ») suivie de deux explications : leur propre haine et l'aversion de leur dieu. En soi, selon nos critères, ces éléments suffisent à classer cet extrait parmi les quasi-Questions, même s'il faut bien reconnaître que c'est un peu maigre. Toutefois, il se trouve que cette information est répétée dans les Propos de table (670D), où Plutarque écrit que «les mages qui se réclament de Zoroastre honorent tout particulièrement le hérisson terrestre, mais ont les rats d'eau en horreur et regardent celui qui en tue le plus grand nombre comme un homme heureux et aimé des dieux $»^{32}$, ce qui confirme que cette pratique curieuse était apparemment répandue et qu'elle avait visiblement une signification religieuse, information que corrobore définitivement un extrait du traité Sur Isis et Osiris (369E-F) qui précise que «les Perses croient en effet que certaines plantes appartiennent au dieu bon, d'autres au démon mauvais; que, parmi les animaux, le chien, les oiseaux et le hérisson, par exemple,

\footnotetext{
${ }^{30}$ Rose (The Roman Questions..., p. 49) le range également parmi ceux qui réutilisent peut-être du matériel provenant des Questions barbares.

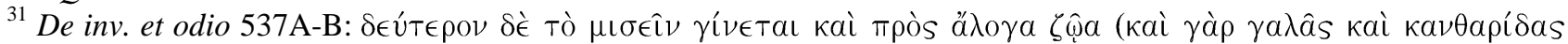

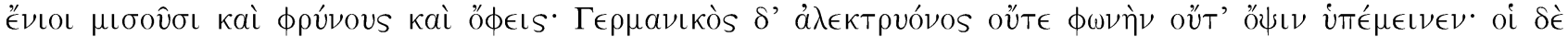

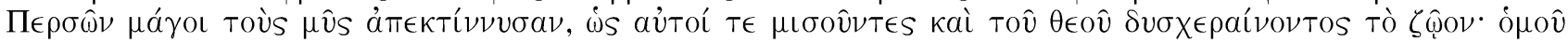

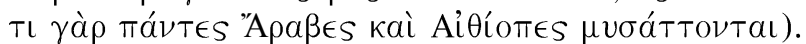

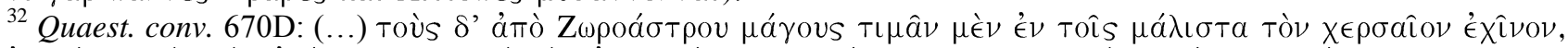

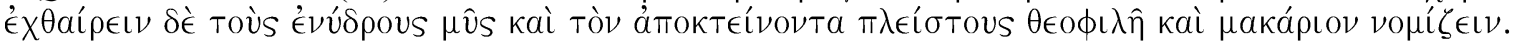


appartiennent au dieu bon, et le rat d'eau à l'autre, au principe mauvais : c'est pour cette raison qu'ils félicitent quiconque en a tué un très grand nombre. ${ }^{33}$ Nous voici donc en présence d'une troisième explication de la haine des Perses pour le rat d'eau : son appartenance au principe mauvais. Qui plus est, nous avons, dans ce dernier extrait, une formule conclusive («c'est pour cette raison que... ») qui, jointe aux informations des deux autres passages, nous autorise à y reconnaître, selon toute vraisemblance, les indices d'une «véritable Question » qui aurait été: «Pourquoi les Perses (ou les mages perses) félicitent-ils quiconque tue un grand nombre de rats d'eau? » et qui, par son sujet (une pratique à connotation religieuse), s'inscrit parfaitement dans la lignée de la majorité des Questions romaines et d'une part importante des Questions grecques.

Cet exemple, par ailleurs, n'est pas isolé : nous avons identifié plusieurs « véritables Questions », douze en tout, à commencer par les trois qui font l'objet de questions en bonne et due forme dans les Propos de table, à savoir les questions 5 et 6 du livre IV (« est-ce par vénération pour le porc ou par aversion que les Juifs s'abstiennent de cette viande?»[669E-671C $]^{34}$ et «quel est le dieu des Juifs? » [671C-672C]), qui donnent toutes deux naissance à des développements de plusieurs pages, et la question 9 du livre VII («délibérer pendant un banquet n'est pas moins une coutume grecque que perse » [714A-D]), où Plutarque s'interroge longuement sur l'origine grecque ou barbare de cette pratique. Deux autres questions véritables se trouvent d'ailleurs également dans les Propos de table: l'une cherche à expliquer pourquoi les prêtres égyptiens s'abstiennent de poisson (729A-C) et rejoint par là l'une des quasi-Questions évoquées plus haut (De Iside 353C-D), ce qui nous invite à fusionner les explications proposées; l'autre s'intéresse aux raisons qui poussent les prêtres égyptiens à s'abstenir de sel (684F-685A) - le sujet est abordé dans le cadre d'une question plus large portant sur le fait que le sel est qualifié par Homère de «divin ». Voici comment Plutarque amène le sujet:

«Ce qui augmentait notre perplexité, c'était que les prêtres égyptiens s'abstiennent absolument de sel, par souci de perfection, au point même de manger leur pain non salé : comment ont-ils pu, si le sel est «aimé des dieux » et « divin », s'imposer cette interdiction? Florus nous pria de laisser les Égyptiens tranquilles et de nous en tenir à la Grèce pour parler de la question. Je répondis que les Égyptiens n'étaient nullement contraires en cela aux Grecs ; car la recherche de la perfection la plus scrupuleuse est incompatible avec l'acte de procréation, avec le rire, avec l'usage du vin et

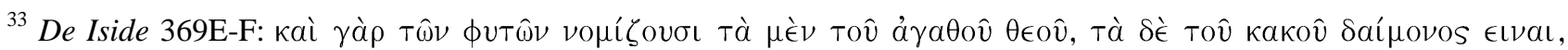

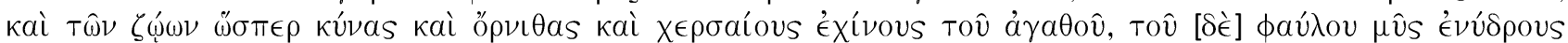

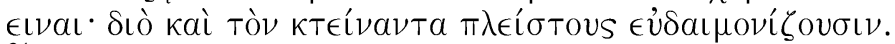

${ }^{34}$ C'est l'un des extraits identifiés par Rose (The Roman Questions..., p. 49) comme reprenant du matériel des Questions barbares.
} 
avec beaucoup d'autres choses habituellement fort désirables; il est donc bien possible que les Égyptiens évitent le sel, dans leur souci de pureté, parce que la chaleur excite les appétits sexuels, comme certains le prétendent; mais on peut supposer aussi qu'ils s'en privent comme du condiment le plus agréable; car le sel a bien l'air de n'être qu'un condiment qui agrémente les autres condiments ; c'est d'ailleurs pourquoi certains l'appellent charitas (« charmes »), parce que de la nourriture nécessaire il fait une nourriture agréable. ${ }^{35}$

Comme on le voit très clairement, la question est posée d'emblée (« comment les Égyptiens ontils pu s'abstenir du sel alors qu'il est divin? ») et deux explications sont avancées : (a) la recherche de la perfection et de la pureté, (b) le rejet de l'agréable. Il convient de noter aussi les formules qui introduisent les alternatives : «il est donc bien possible que... » et «mais on peut supposer aussi que... ». Elles sont tout à fait dans la lignée des hypothèses multiples évoquées dans les Questions grecques et les Questions romaines. A cela s'ajoute que cette question de l'abstinence du sel est abordée également dans le traité Sur Isis et Osiris. En 352F, Plutarque explique :

«Les prêtres ont une telle répugnance à l'égard des excréments de toute nature que, non contents de s'abstenir de la plupart des légumineuses et de la viande de mouton et de porc, qui produisent beaucoup de résidus, ils s'interdisent aussi, durant tout le temps où ils doivent rester purs, le sel dans les aliments, et cela, entre toutes raisons qu'ils donnent de cet usage, parce qu'il excite l'appétit et pousse aux excès de boisson et de table. L'opinion émise par Aristagoras, qui explique l'impureté du sel par tous les animalcules qui se laisseraient prendre et mourraient dans ses cristaux lors de leur formation est une absurdité. ${ }^{36}$

Même si la question n'est pas formulée de façon explicite, on retrouve le même contexte que dans l'extrait précédent : l'explication de cette abstinence est toujours la question de la pureté, mais on voit bien que Plutarque approfondit ou plutôt élargit la question. A noter aussi qu'il évoque plusieurs raisons ( $\pi \lambda \epsilon$ íovas aiтías) avancées par les prêtres eux-mêmes et qu'il fait une digression critique à

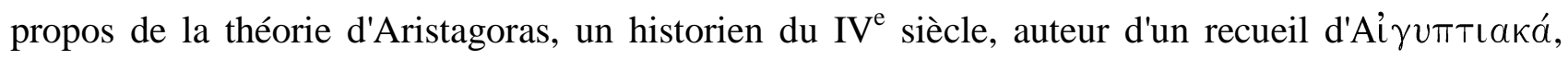

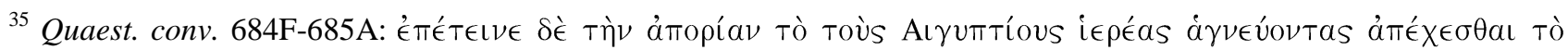

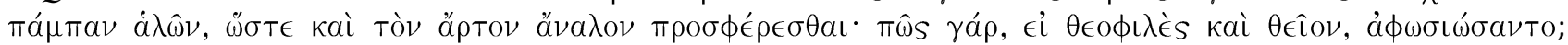

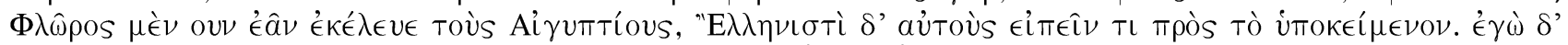

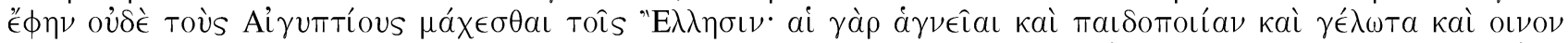

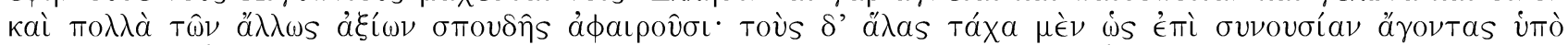

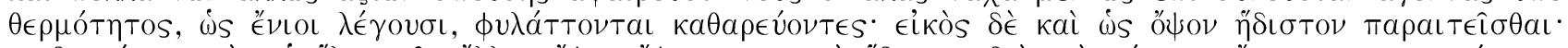

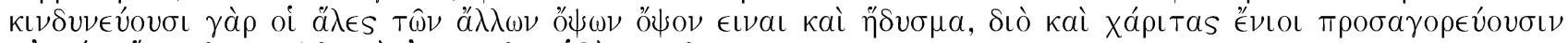

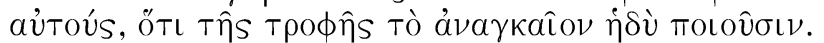

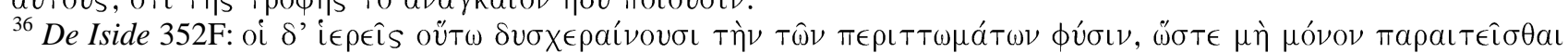

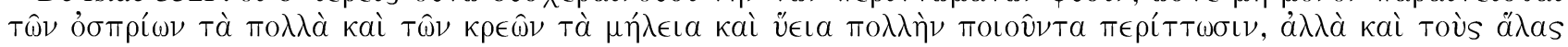

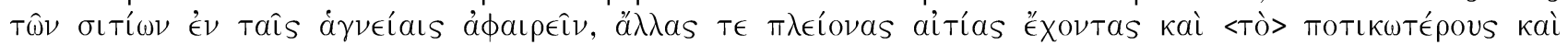

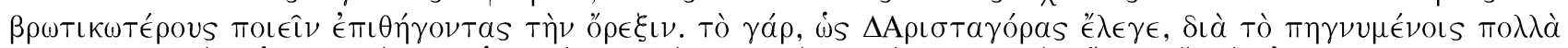

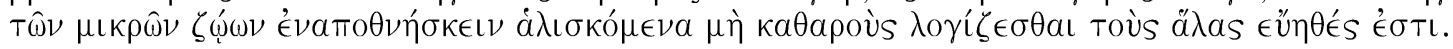


deux indices supplémentaires de l'existence d'une question. Mais ce n'est pas tout : la question est encore abordée une autre fois dans le même traité ; en 363E-F, Plutarque écrit :

«Les Égyptiens croient en effet que l'Est est la face du monde, le Nord sa droite et le Sud sa gauche ; comme le Nil vient du Sud et qu'il est englouti au nord par la mer, il est normal de dire qu'il prend naissance dans les régions de gauche et trouve sa fin dans les régions de droite. Pour cette raison, les prêtres abominent la mer et appellent le sel «l'écume de Typhon »; le sel fait partie des aliments qui ne doivent pas figurer sur leur table, et ils n'adressent pas la parole aux pilotes parce que ceux-ci vivent en mer et vivent de la mer. C'est une des principales raisons de leur aversion à l'égard du poisson... » ${ }^{37}$

La question est ici élargie à la notion de rejet de la mer, mais elle offre, à propos de l'abstinence de sel, une nouvelle explication qui vient compléter les précédentes. Il ne semble pas absurde d'imaginer que toutes ces explications réunies aient pu faire l'objet d'une seule et même Question barbare. D'ailleurs, Plutarque termine le dernier extrait en disant : «Fermons là cette parenthèse: ces considérations débordent notre présent propos. ${ }^{38}$ Cela semble impliquer qu'il ait pu ou voulu en parler ailleurs. Alors pourquoi ne pas imaginer que c'était dans les Questions barbares ? L'hypothèse semble plausible, ce d'autant plus qu'à la fin d'une autre des «véritables Questions » que nous avons identifiées dans le traité Sur Isis et Osiris, qui explique pourquoi les prêtres d'Isis se rasent et s'habillent de lin (352C-E), Plutarque conclut par ces mots : «Mais j'aborde ailleurs ce sujet. » ${ }^{39}$ Or cet ailleurs n'a pas été identifié de façon satisfaisante par les éditeurs ou commentateurs de l'extrait $^{40}$.

$\mathrm{Au}$ terme de notre essai de reconstitution, il apparait que les indices ne manquent pas pour déceler les traces de « véritables Questions » dans l'œuvre de Plutarque. Il est gênant, cependant, de constater que les douze «véritables Questions» identifiées proviennent toutes soit des Propos de table soit du traité Sur Isis et Osiris, et qu'il en est de même pour les «quasi-Questions », dont

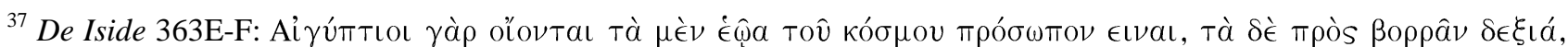

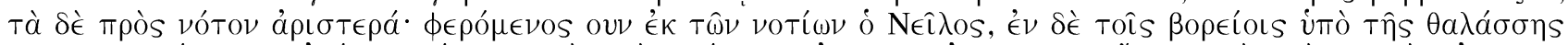

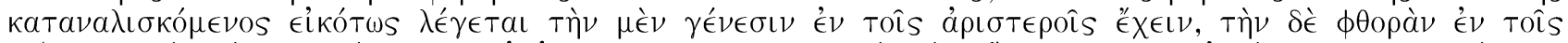

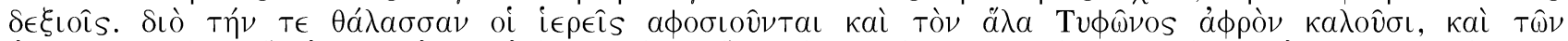

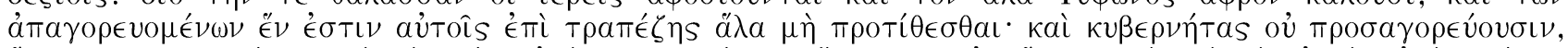

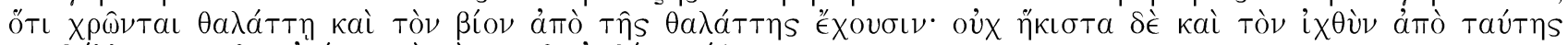

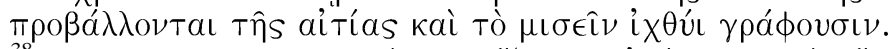

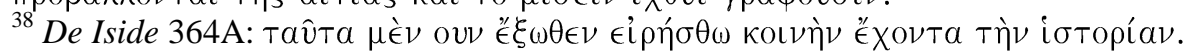

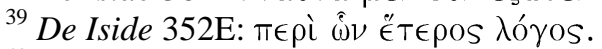

${ }^{40}$ Il est vrai qu'il n'est pas clair à quel élément du contexte la phrase de Plutarque fait référence. C. Froidefond (Plutarque, Oeuvres morales, tome V, $2^{\mathrm{e}}$ partie : Isis et Osiris, Paris, CUF, 1988, p. 181 n. $3=$ p. 256) renvoie à Quaest. rom. 286E (sur les raisons de s'abstenir de légumes) et à Quaest. conv. 642C (sur la vermine) ; voir aussi T. Hopfner, Plutarch, Über Isis und Osiris, Prague, 1940, p. 60-64 ; F.C. Babbitt, Plutarch's Moralia, V, London-Cambridge, MA (Loeb), 1957, p. 14 ; J.G. Griffiths, Plutarch's De Iside et Osiride, Cardiff, 1970, s'abstient de tout renvoi.
} 
quatre sur cinq se trouvent dans le Sur Isis et Osiris. On pourrait donc penser que ces questions ont été écrites pour l'une ou l'autre de ces deux œuvres et qu'elles n'avaient pas du tout leur place dans les Questions barbares $^{41}$. Toutefois, comme nous avons essayé de le montrer, c'est la complémentarité des explications qui, à nos yeux, représente un argument sinon décisif, du moins plausible d'une reprise, sous une forme plus élaborée, des questions abordées de façon partielle dans les deux traités mentionnés. Par ailleurs, si notre récolte d'indices permettant de reconstituer des questions barbares concrètes peut sembler décevante, il convient de préciser, d'une part, que ce fait était prévisible, puisque même pour les Questions grecques et les Questions romaines, les échos dans le reste du corpus plutarchéen sont somme toute peu nombreux ${ }^{42}$; d'autre part, il nous semble que les résultats obtenus, en particulier la variété des pratiques barbares abordées, sont néanmoins suffisants pour affirmer que Plutarque avait un intérêt marqué pour les coutumes et les traditions des barbares et qu'il est donc parfaitement plausible qu'il les ait traitées de façon plus systématique dans

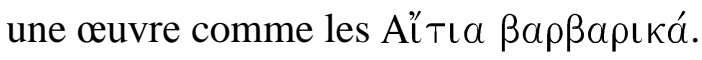

Il ne nous échappe pas que la fragilité de nos résultats rend vain tout essai de synthèse sur la nature des Questions barbares. En guise de conclusion, qu'on nous permette seulement quelques constatations d'ordre général. Les intérêts de Plutarque semblent avoir concerné en premier lieu les pratiques religieuses des barbares, puisque celles-ci occupent la moitié des questions identifiées, comme c'est aussi le cas, du reste, pour les Questions romaines. Aucun autre thème majeur ne semble se dégager, les pratiques décrites étant au contraire extrêmement variées. Seule la question des rites funéraires et des expressions du deuil se démarque quelque peu - sept extraits s'y rapportent ${ }^{43}$-, ce qui ne surprend guère quand on sait que Plutarque partage l'avis (le préjugé ?) de nombreux Grecs au sujet, notamment, de l'excès des barbares dans le deuil ${ }^{44}$. La même variété se retrouve à propos des peuples nommés : les Égyptiens et les Perses occupent certes une place prépondérante, mais au total ce ne sont pas moins de dix-sept peuples barbares, orientaux comme occidentaux, qui apparaissent dans nos extraits ${ }^{45}$. Cette constatation rend caduque, à nos yeux, l'hypothèse selon laquelle les Questions barbares seraient des notes de lecture faites à l'occasion de

\footnotetext{
${ }^{41}$ Voir notamment l'avis de Harrison, «Problems... », p. 197, qui estime que la structure souple des Propos de table permettait à Plutarque d'y insérer des questions qui n'avaient pas leur place dans d'autres recueils d'aítíaı.

${ }^{42}$ Pour les Questions barbares, il convient de signaler que les traces de cette œuvre se retrouvent en très forte majorité dans les Moralia; seuls quatre extraits, qui plus est de simples «Questions potentielles », proviennent des Vies.

${ }^{43}$ Consol. ad Apoll. 113A-B ; De Iside 352 B ; An vitiositas suff. 499C et 499D (2x) ; De sera num. vind. 557D ; De esu carn. 996E.

${ }^{44}$ Voir nos remarques dans Plutarque et les barbares..., p. 232-233.

${ }^{45}$ Ce sont les Égyptiens (18 mentions), les Perses (12), les Scythes (3), les Juifs (3), les Gaulois (2), les Éthiopiens (2), les Syriens (2) et, avec une mention, les Indiens, les Hyrcaniens, les Mèdes, les Assyriens, les Libyens, les Thraces, les Bretons et les habitants du Nord de l'Italie ; à cette liste s'ajoutent quatre cas indéterminés (où il est question des barbares en général). À noter aussi que huit questions se rapportent à des pratiques de femmes.
} 
la rédaction de la Vie d'Artaxerxès et du traité Sur Isis et Osiris: ces deux œuvres ont incontestablement joué un rôle important, mais ne sont visiblement pas les seules sources d'information de Plutarque. Ces dernières sont toutefois difficiles à identifier, entre autres parce que

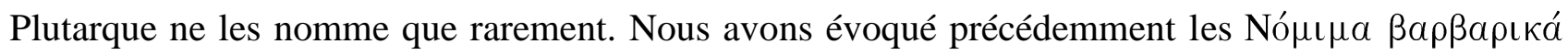
d'Aristote, qui ont vraisemblablement été une source importante. Plusieurs «questions » semblent remonter à Hérodote (Conj. praec. 142C ; An virt. doc. possit 440A ; An vitiositas suff. 499D), une autre fait mention d'Aristagoras, auteur d'Aỉyutrıká (De Iside 352F-353A), et pour d'autres

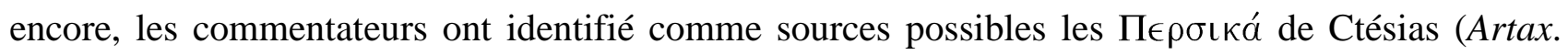

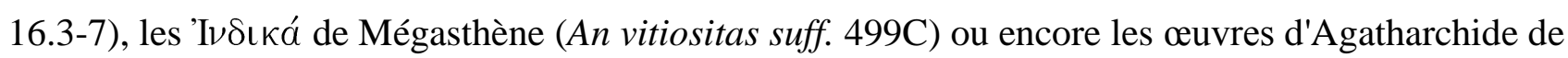
Cnide (De comm. notit. 1064B) et de Chrysippe (Consol. ad Apoll. 113A-B) ${ }^{46}$. Sans doute Plutarque a-t-il pris ses informations également chez l'historien Juba, roi de Maurétanie, qu'il utilise fréquemment dans les Questions romaines et dont l'œuvre, les "O comparaisons entre les pratiques des Romains et celles d'autres peuples ${ }^{47}$. N'oublions pas non plus que Plutarque a lui-même séjourné en Égypte ${ }^{48}$ et qu'il a vraisemblablement été en contact avec les cultes isiaques, bien attestés dans sa Béotie natale, en particulier à Chéronée ${ }^{49}$ : il a donc pu avoir, dans certains cas, des informations de première main. Ses sources, toutefois, semblent avoir été avant tout littéraires, ce qui n'a rien d'étonnant de la part d'un homme si cultivé et d'un compilateur aussi assidu que curieux. Ses Questions barbares ne nous semblent pas, pour autant, n'avoir été qu'un ramassis de curiosités, comme on a pu le prétendre à propos des Questions grecques et des Questions romaines. La prépondérance de pratiques liées à la religion est au contraire le signe d'une certaine cohérence, du reste en accord avec le caractère général des Questions romaines. À quel public Plutarque les destinait-il? Sans doute aux mêmes lecteurs que pour les Questions grecques et les Questions romaines, à savoir ce public de gens cultivés que comptaient l'entourage et le cercle d'amis de Plutarque, tant grecs que romains, ces $\phi \iota \lambda o ́ \lambda o \gamma o \iota$ au sens noble qui, sans être des érudits, se passionnaient pour toutes sortes de questions et se montraient intéressés par les temps anciens

\footnotetext{
${ }^{46}$ Ce ne sont que quelques exemples. Tout comme pour les Questions grecques et les Questions romaines, les sources mobilisées par Plutarque pour la rédaction des Questions barbares ont dû être abondantes ; il suffit de penser aux très nombreux auteurs qu'il a utilisés pour la rédaction du traité Sur Isis et Osiris, cf. Froidefond, Plutarque..., CUF, p. 45-

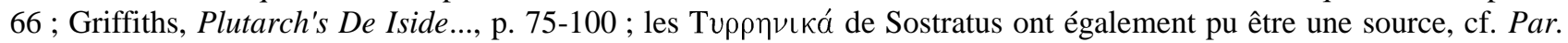
min. 312C-D.

47 Voir Rose, The Roman Questions..., p. 20-27; Van der Stockt, «Plutarch's Use of Literature.. », p. 283-287 ; Boulogne, Plutarque...,CUF, p. 103 ; Nouilhan, Pailler, Payen, Plutarque..., p. 26.

${ }^{48}$ Ziegler, Plutarchos..., col. 654 ; Sirinelli, Plutarque..., p. 46-51.

${ }^{49}$ Voir Hani, La religion égyptienne..., p. 8-12 ; F. Dunand, Le culte d'Isis dans le bassin oriental de la Méditerranée, II: le culte d'Isis en Grèce, Leyde, 1973, p. 29-39; L. Bricault, Recueil des inscriptions concernant les cultes isiaques, vol. 1, Paris, 2005, p. 57-102, et idem, Atlas de la diffusion des cultes isiaques, Paris, 2001, p. 10-13. Je dois les deux dernières références au professeur Denis Knoepfler, que je remercie vivement.
} 
$(\phi \iota \lambda a ́ \rho \chi a \circ \iota)^{50}$. Il semble donc assez naturel que Plutarque ait adopté, dans les Questions barbares, le point de vue d'un Grec s'étonnant de certaines pratiques barbares déviant de sa propre culture de référence. Toutefois, alors que les œuvres de Plutarque comportent plus d'une remarque désobligeante à l'égard des barbares ${ }^{51}$, dans les extraits des Questions barbares que nous avons cru pouvoir identifier, cela ne semble pas être le cas. Tout porte à croire, au contraire, que Plutarque ait voulu transmettre de façon objective à ses contemporains les connaissances qu'on pouvait avoir sur les pratiques des peuples barbares et d'en chercher les causes sans porter sur elles de jugements de valeur, mais en les intégrant dans la description plus large d'un monde où se côtoient les Grecs, les Romains et les barbares ${ }^{52}$.

Thomas SCHMIDT

Université Laval - Québec

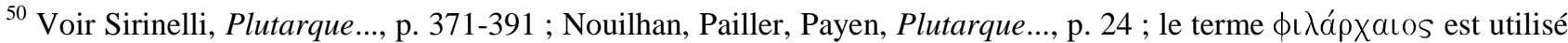
notamment à propos de Florus (Quaest. conv. 702D) et de Saturninus (Adv. Colot. 1107E).

${ }^{51}$ Voir notre étude Plutarque et les barbares... (passim). Par ailleurs, les Questions romaines ne sont pas exemptes de préjugés face aux Romains, comme le rappelle Boulogne, «Les "Questions romaines"... », p. 4702. Sur les rapports complexes entre hellénisme et romanité dans les Questions grecques et les Questions romaines, voir Preston, « Roman questions... », p. 97-109.

${ }^{52}$ Dans cette perspective, il serait logique de situer la date de rédaction des Questions barbares en même temps que les Questions grecques et les Questions romaines, deux œuvres contemporaines qui furent vraisemblablement rédigées vers 100-110 (cf. Harrison, « Problems... », p. 197-198 ; Boulogne, Plutarque..., CUF, p. 104-105 et p. 182), mais aucun élément ne permet de l'affirmer avec certitude.
} 